\title{
A Change-Detection Algorithm Enabling Intelligent Background Maintenance
}

\author{
Luigi Di Stefano ${ }^{1,2}$, Stefano Mattoccia ${ }^{1,2}$, and Martino Mola ${ }^{1,2}$ \\ 1 Department of Electronics Computer Science and Systems (DEIS) \\ University of Bologna, Viale Risorgimento 2, 40136 Bologna, Italy \\ 2 Advanced Research Center on Electronic Systems 'Ercole De Castro' (ARCES) \\ University of Bologna, Via Toffano 2/2, 40135 Bologna, Italy \\ \{ldistefano, smattoccia, mmola\}@deis.unibo.it
}

\begin{abstract}
We have recently proposed a change-detection algorithm based on the idea of incorporating into the background model a set of simple low-level features capable of capturing effectively "structural" information. In this paper we show how this algorithm can naturally interact with the higher-level processing modules found in advanced videobased surveillance systems so as to allow for flexible and intelligent background maintenance.
\end{abstract}

\section{Introduction}

Advanced videosurveillance systems typically include as the first image analysis step a change-detection algorithm aimed at segmenting out the interesting regions from a background. Then, higher level processing modules, such as tracking, classification and interpretation modules, process the output of the change detection algorithm to attain the required degree of scene understanding.

Most change-detection algorithms rely on the principle of background subtraction: a background model is compared to the current image in order to mark as foreground those pixels that exhibit a significant difference with respect to the corresponding background pixels. The main difficulty associated with change-detection is not the background subtraction step, but instead the maintenance of a background model that follows correctly the changes of the reference scene. These can be grouped into illumination changes and changes due to objects. The latter occur when an object is introduced into or removed from the reference scene. If a foreground object stops a decision should be taken on whether and when it is more appropriate to include its appearance into the background model. Similarly, if a background object starts moving, its absence in the previously occupied image region is detected as a bogus blob (usually known as ghost). In this case, the elimination of the ghost is typically desirable and can be achieved by updating the background in the region previously occupied by the object.

We have recently proposed a novel change-detection approach [1] that relies on a background model very robust with respect to illumination changes, so 
that in principle it needs to be updated only to handle the insertion/removal of objects. In this paper we show how the changes due to objects can be dealt with effectively and intelligently by exploiting an interaction between the changedetection level and the higher processing levels found in advanced video-based surveillance systems.

\section{Previous Work and Proposed Approach}

Among the change detection algorithms relying on the background subtraction principle, the statistical approach is the most widely adopted one $2,3,4,56]$. With this approach some features are used to represent the background pixels (e.g. RGB components, hue, luminance, gradient) and modelled by a probability distribution. A pixel of the current image is then classified as foreground if the observed features are not coherent with the associated probability distribution. Background maintenance consists typically in updating the parameters of the probability distributions based on the last observed images. As regards the background subtraction principle, the method proposed in [6] is the most similar to ours since image gradient is used to achieve robustness with respect to illumination changes and the combination of gradient and colour information is done at region-level. However, we extract gradient information at a reduced resolution to significantly improve robustness and explicitly exploit illuminationinsensitivity within the background maintenance process. The idea of exploiting an interaction between the change-detection level and higher-level modules can be found also in [5]. Yet, this method is much more complex since it relies on combining in a Mixtures of Gaussians framework colour information with the depth measurements provided by a stereo system.

Excluding [5], in the above mentioned algorithms a foreground object is immediately and gradually included into the background as soon as it stops moving so that, after a certain time interval, it will be perceived as background. Similarly, a ghost will be absorbed into the background according to the same dynamics. It is worth pointing out that this common strategy allows the changedetection algorithm to recover from persistent false positive errors and that this is of fundamental importance for proper long-term functioning of the algorithm. However, this strategy relies on a user-selectable time constant that determines the time needed for a pixel repetitively classified as foreground to become part of the background. If the time constant is fast false positives are absorbed very quickly into the background model but slowly moving objects may corrupt the background. Conversely, if the time constant is slow, slowly moving objects are correctly detected but recovering from false positive errors takes a long time.

Ideally, if the background model were based on features invariant to illumination changes the above problem would be removed since the background model should be updated only to accommodate the changes due to objects. Though it seems impossible to find low-level features invariant to every kind of illumination changes, it is possible to devise low-level features resulting robust with respect to many illumination changes occurring in practise. Starting from these consid- 
erations, we have devised a set of very simple low-level features, referred to as image structure, that have proven to be very robust with respect to illumination variations. By including these features into the background model we have obtained a novel change detection algorithm that approximates satisfactorily the ideal behaviour outlined previously. Since in principle our algorithm relies on a background model that needs to be updated only to handle "high-level" events, namely insertion/removal of objects, it naturally holds the potential to interact with higher-level processing modules that may control the background maintenance process flexibly and intelligently. Thus, our algorithm has been designed to support easily this kind of interaction: it can accept a binary mask that controls the inclusion/removal of objects into the background model.

The results reported in our previous paper [1] were obtained running the algorithm in "stand-alone" mode (i.e. without any feedback from higher-level modules). Here we discuss some new results that demonstrate how the algorithm can usefully interact with a simple higher-level module that tracks and classifies the blobs provided by the change-detection level.

\section{The Change-Detection Algorithm}

Given a $w \times h$ grey-level image, $I$, the first step needed to extract image structure, consists in obtaining a reduced-resolution image, $R[I]$. Let $\delta$ be a scale factor, then $R[I]$ is a $\frac{w}{\delta} \times \frac{h}{\delta}$ image defined as:

$$
R[I](x, y)=\frac{1}{\delta^{2}} \sum_{j=0}^{\delta-1} \sum_{k=0}^{\delta-1} I(\delta \times x+j, \delta \times y+k)
$$

Then we obtain two additional $\frac{w}{\delta} \times \frac{h}{\delta}$ images defined as:

$$
\begin{aligned}
& D_{x}[I](x, y)=R[I](x+1, y)-R[I](x, y), \\
& D_{y}[I](x, y)=R[I](x, y+1)-R[I](x, y) .
\end{aligned}
$$

$D_{x}[I], D_{y}[I]$ are simply the horizontal and vertical derivatives of the reducedresolution image $R[I]$, and the pair $D_{x}[I], D_{y}[I]$ forms the structure of the original grey-level image $I$. To obtain structure in the case of colour images we simply apply the described transformation to each of the three RGB channels. In this manner we obtain six $\frac{w}{\delta} \times \frac{h}{\delta}$ images: $D_{x, r}[I], D_{x, g}[I], D_{x, b}[I], D_{y, r}[I], D_{y, g}[I]$, $D_{y, b}[I]$ (the second subscript identifies the colour channel). We have found that in real video sequences the structure variations produced by illumination changes are usually much smaller than those caused by true "structural" changes of the scene. However, a change detection process based solely on structure would detect changes with a very low resolution, yielding inaccurate blobs. To overcome this problem we adopt a background model made out of two separate parts: the first is the structure of the scene without moving objects while the second is simply a colour image of the reference scene. The first part of the model will be referred to as background structure, its components indicated as $B D_{x, r}, B D_{x, g}$, 
$B D_{x, b}, B D_{y, r}, B D_{y, g} B D_{y, b}$. Since its variations can be largely ascribed to objects, its updating is focused on handling this type of changes, with a simple mechanism allowing a feedback from a higher-level processing module. The second part of the model will be referred to as background image, its components indicated as $B_{r}, B_{g}, B_{b}$. It will provide the algorithm with the capability of detecting blobs at the highest possible resolution.

Given the described background model, at each new frame the detection process operates at both the structure and image level; then, the detection results are combined adequately to obtain the final output. Before starting the detection process, our algorithm activates a simple "bootstrap" process aimed at estimating the initial data to be included into the background model.

Structure-Level Detection. We compare the structure of the current frame, $I$, with the background structure by building up two delta-structure images associated respectively with the $x$ and $y$ directions:

$$
\Delta S_{d}[I](x, y)=\max _{c h \in\{r, g, b\}}\left\{\left|B D_{d, c h}(x, y)-D_{d, c h}[I](x, y)\right|\right\}, d \in\{x, y\}
$$

Then, choosing a suitable threshold value, $t_{s}$, and recalling equation (2), we can observe that if $\Delta S_{x}[I]>t_{s}$ at structure element $(x, y)$, then a foreground object occupies (or a background object leaves) the image region associated with structure element $(x, y)$ or $(x+1, y)$, or both. Similarly if $\Delta S_{y}[I]>t_{s}$ at structure element $(x, y)$, the structure change could be located at element $(x, y)$ or $(x+1, y)$, or both. Therefore, we define the $\frac{w}{\delta} \times \frac{h}{\delta}$ binary image $S_{\text {mask }}$ containing the structure-level detection results as:

$$
S_{\text {mask }}(x, y)=\left\{\begin{array}{rr}
1, & \text { if }\left(\Delta S_{x}[I](x, y)>t_{s}\right) \\
\left(\Delta S_{y}[I](x, y)>t_{s}\right) & \vee\left(\Delta S_{x}[I](x-1, y)>t_{s}\right) \vee \\
0, & \text { otherwise }
\end{array}\right.
$$

Image-Level Detection. In this case the detection step is simpler and consists in computing the difference between $I$ and the background image. Hence, calling $I_{r}, I_{g}, I_{b}$ the colour channels of the current frame and $t_{p}$ a new threshold value, we define the $w \times h$ binary image $I_{\text {mask }}$ containing the image-level detection results as:

$$
I_{\text {mask }}(x, y)=\left\{\begin{array}{l}
1, \text { if } \max _{c h \in\{r, g, b\}}\left\{\left|I_{c h}(x, y)-B_{c h}(x, y)\right|\right\}>t_{p} \\
0, \text { otherwise. }
\end{array}\right.
$$

Combination of the Detection Results. The information contained in $S_{\text {mask }}$ is used to decide whether or not each of the blobs detected in $I_{\text {mask }}$ is valid. The validation is done by labelling the connected components of $I_{\text {mask }}$ and erasing those not intersecting at least one structure-element marked with 1 in $S_{\text {mask }}$. The result of the combination step is a $w \times h$ binary image, Mask, that contains only the blobs associated with objects changing image structure. 
Updating of the Background Structure. If at a certain structure element the difference between the background structure and the current image structure is persistently above the threshold $t_{s}$, the system estimates the value that should be assigned to the element to absorb the change. The estimation process consists in observing the temporal behaviour of the above-threshold structure element until it exhibits a stable value. Only when this occurs the estimated value may be used to update the background structure. In fact, the actual updating must be enabled explicitly by a feedback information coming from a higher-level module. This consists of a binary image $F B$, as large as $M a s k$, in which the higher-level module should redraw the blobs associated with "interesting" objects. Then, the structure elements intersecting at least one blob drawn in $F B$ will not be updated, even though a stable value might be available. Conversely, if a structure element exhibiting a persistent change does not intersect any blob drawn in $F B$ and an estimated value is available, the estimated value is copied into the background structure.

Updating of the Background Image. Indicating the background image before and after the updating as $B^{t}$ and $B^{t+1}$ respectively, and considering the red channel, the updating rule is given by:

$$
B_{r}^{t+1}(x, y)=\left\{\begin{array}{l}
B_{r}^{t}(x, y)+\eta, \quad \text { if }(\operatorname{Mask}(x, y)=0) \wedge\left(B_{r}^{t}(x, y)<I_{r}(x, y)\right) \\
B_{r}^{t}(x, y)-\eta, \quad \text { if }(\operatorname{Mask}(x, y)=0) \wedge\left(B_{r}^{t}(x, y)>I_{r}(x, y)\right) \\
B_{r}^{t}(x, y), \quad \text { otherwise }
\end{array}\right.
$$

where $\eta$ is a constant value. The same rule is used also for the green and blue channels.

\section{Experimental Results}

The experiments are aimed at demonstrating how our change detection algorithm can usefully interact with a simple tracking-classification module. The scene is a parking lot, where we can observe pedestrians and vehicles approaching to the parking lot or leaving the scene after a prolonged stop. The tracking system is similar to that described in [7]: it tracks the blobs provided by the change detection module by establishing frame-by-frame correspondences on the basis of distance measurements and handles blob merging-split events by means of a set of heuristic rules. Many other tracking systems (e.g. 89104]) rely on a blob-based approach, adopting different strategies to handle blob mergingsplit events. The feedback action between the change detection algorithm and the higher-level module is aimed at facilitating the tracking task by avoiding as many blob-merging events as possible and by not including into the background the objects that stay still for a short time frame.

In a parking lot scenario, if we use a traditional change detection algorithm with a slow time constant, every time a car stops we observe a motionless blob that could merge with other objects moving in the same area, as could be the passengers getting off the parked car. Moreover when a car moves away after a stop, a ghost is produced and its blob is a potential source of merges with 
the blob of other objects moving around. These two problems could be partially solved by adopting a fast time constant. Unfortunately, with this choice if a person temporarily stops moving, he will be no longer detected and will soon produce a ghost when walking away. Moreover, in a parking lot the detection of a motionless person is typically desirable to discover suspicious activities.

The above problems can be dealt with effectively by exploiting our algorithm's capability to receive feedback information from a higher-level module. Basically, the change-detection algorithm should be controlled so as to continuously detect persons and ceasing rapidly the detection of still cars and ghosts by absorbing them quickly into the background. Recalling section 3 , this can be obtained by always redrawing in FB all the detected blobs but still cars and ghosts. To proceed in this way, a classifier capable of recognising still cars and ghosts must be built on top of the tracking module. The very simple classifier adopted in our experiments is based on the following rules:

1. A tracked object having a motionless blob larger than a fixed threshold is a still car.

2. After a blob splits, if one of the resulting blobs is motionless and larger than a fixed threshold while the other resulting blobs are moving, then the motionless blob is the ghost of a car moved away.

The first rule allows fast insertion of all still cars into the background. In these cases when successively the passengers get off the parked car, a ghost may appear inside the vehicle. These kind of ghosts can be detected by means of the following additional rule:

3. If a blob appears within the region associated with a previously absorbed still car and successively splits into still and moving blobs, then the moving blobs are classified as passengers and the still ones as ghosts.

Figure 1 shows several frames from a sequence with a parking vehicle. Each snapshot contains on the left the tracking results (a labelled bounding-box superimposed on the original image) and on the right the change-detection output (a binary image). In snapshots (a) and (b) a car approaching to a parking lot is detected and easily tracked until it stops. The time elapsed between the first two snapshots is 4,88 seconds (as indicated in the figure). Now, our simple classifier applies rule 1 and recognise the tracked object as a still car, thus enabling its quick inclusion into the background. In fact, after 6 seconds (snapshot c), the car is still detected, but in the successive frame (snapshot d) its blob disappears instantaneously since the car has been included into the background model. As e result, in (e) only the pedestrian passing-by the parked car is detected. Hence, the tracking of the pedestrian has been significantly facilitated by avoiding a merge with the parked car. In (f), after 10,64 seconds, the driver starts getting off the car. The associated blob is tracked and, as expected, a ghost is produced inside the car. When the driver splits away from the ghost, the classifier recognize the motionless blob as a ghost (snapshot $\mathrm{g}$ ) by applying rule 3 , and consequently after only 1,4 seconds, the ghost is included into background and no longer detected (snapshot $\mathrm{h}$ ). Now the driver's blob is the only one to be detected and hence its tracking is straightforward. In (i) the driver 

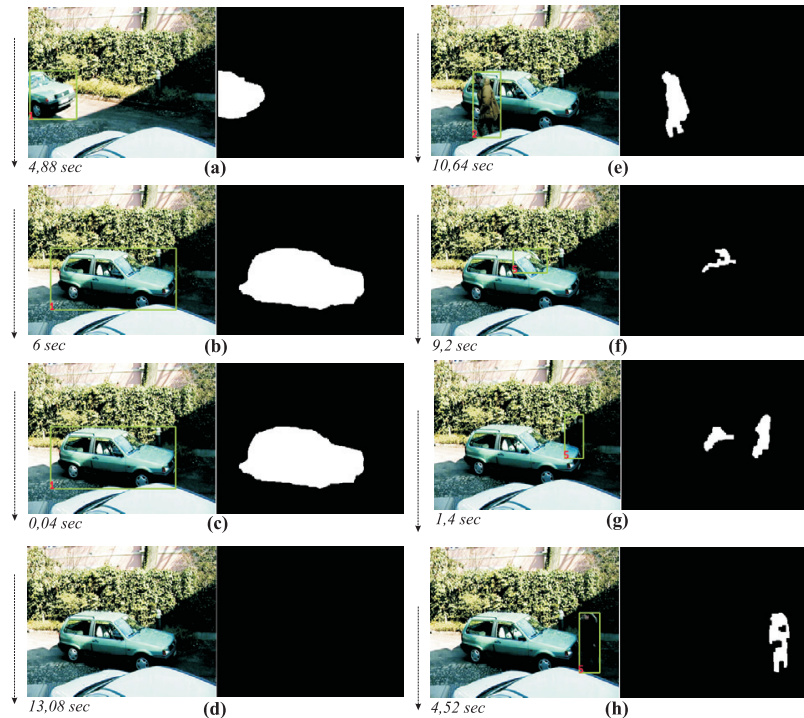

(e)

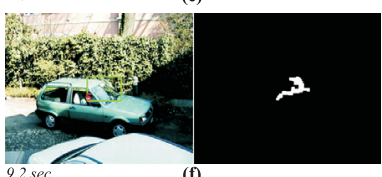

(f)

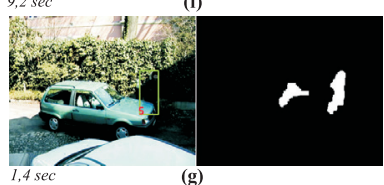

(g)

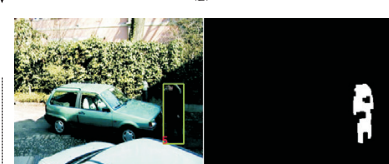

(h)
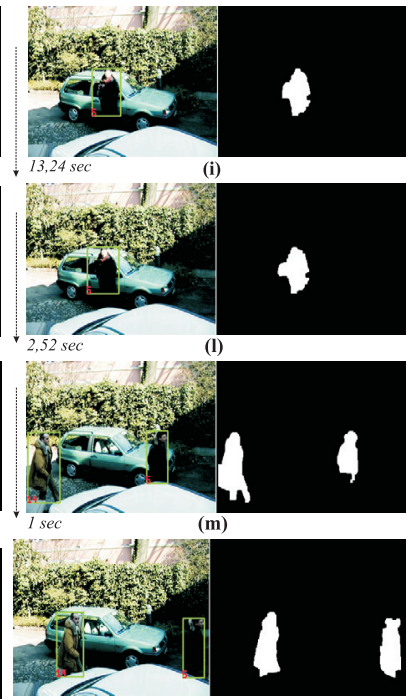

(n)

Fig. 1. A sequence with a parking vehicle.

stops walking and rests completely motionless in front of the car for more than 13 seconds. Though motionless for a long time frame, his blob is continuously detected, and hence straightforwardly tracked since the classifier point it out as an "interesting" object (i.e. not a still car nor a ghost), thus avoiding its inclusion into the background. When successively the driver walks away no ghost is produced, as can be seen in $(\mathrm{m})$ where another pedestrian passes through the region previously occupied by the driver.

Figure 2 shows some frames from a sequence taken some minutes after the previous one. Initially, the background model contains the parked car, that subsequently will leave the parking lot. In snapshot (a) the driver enters the scene and in (b) gets into the car. So far only the driver has been detected, but when the car stars moving (snapshot c), the blob shown in the output is originated by a real object (i.e. the car) as well as its ghost. In snapshot (d) a split event occurs and in (e) the classifier applies rule 2 to recognize the motionless blob as a ghost. Consequently, after a few seconds (snapshot $\mathrm{f}$ and $\mathrm{g}$ ), the still car no longer belongs to the background and hence the ghost is instantaneously eliminated from the output, as can be seen in (h) where a pedestrian is correctly detected and straightforwardly tracked while walking through the region previously occupied by the ghost.

\section{Conclusion}

The proposed change detection algorithm relies on a background model that has been designed to receive feedback information from higher-level processing mod- 


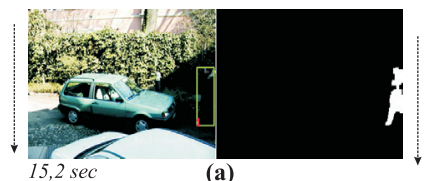

(a)



(b)

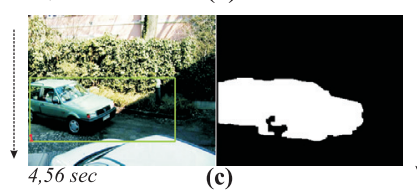

(c)

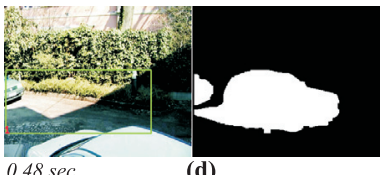

(d)



(e)

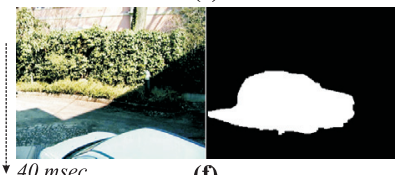

(f)

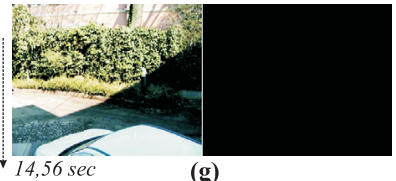

(g)

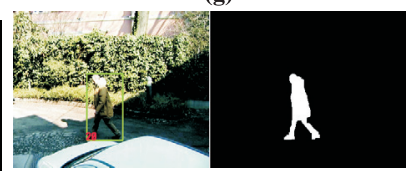

(h)

Fig. 2. A sequence with a leaving vehicle.

ules, thus allowing for flexible and intelligent control of the insertion/removal of objects into the background model. This can be deployed to attain a changedetection output optimised with respect to the specific requirements of the addressed application.

We have demonstrated this capability considering a parking lot and showing that the change-detection output can be optimised so as to facilitate significantly the blob-tracking task by minimising the merges between still cars, ghosts and persons as well as by enabling continuous detection of still persons. This has been achieved by deploying the feedback action from the tracking-classification level to the change-detection level so as to handle properly the inclusion into the background of the tracked objects classified respectively as persons, still cars and ghost. Finally, we point out that the very simple system used in our experiments could be employed in a classical change-triggered Digital Video Recording application. In such a case, unlike a conventional change-detector, our system would trigger the recording only when a car is moving or a person is present, thus storing only the true relevant frames.

\section{References}

1. Di Stefano, L., Mattoccia, S., Mola, M.: A change detection algorithm based on structure and color. In: Int. Conf. on Advanced Video and Signal Based Surveillance. (2003)

2. Wren, C., et al: Pfinder: Real-time tracking of the human body. IEEE PAMI 19 (1997)

3. Haritaoglu, I., Harwood, D., Davis, L.: W4 who? when? where? what? a real time system for detecting and tracking people. In: Int. Conf. on Automatic Face and Gesture Recognition. (1998) 
4. Stauffer, C., Crimson, W.: Adaptive background mixture models for real-time tracking. In: Int. Conf. on Computer Vision and Pattern Recognition. (1999) 246252

5. Harville, M.: A framework for high-level feedback to adaptive, per-pixel, mixtureof-gaussian background models. In: European Conf. on Computer Vision. (2002)

6. Javed, O., Shafique, K., Sha, M.: A hierarchical approach to robust background subtraction using color and gradient information. In: Workshop on Motion and Video Computing. (2002)

7. Di Stefano, L., Mola, M., Neri, G., Viarani, E.: A rule-based tracking system for video surveillance applications. In: Int. Conf. on Knowledge Based Engineering Systems (KES). (2002)

8. McKenna, S., Jabri, S., Duric, Z., Rosenfeld, A., Wechsler, H.: Tracking groups of people. Computer Vision and Image Understanding 80 (2000) 42-56

9. Rosales, R., Scarloff, S.: Improved tracking of multiple humans with trajectory prediction and occlusion modelling. In: Int. Conf. on Computer Vision and Pattern Recognition. (1998)

10. Senior, A., Hampapur, A., Ying-Li, T., Brown, L., Pankanti, S., Bolle, R.: Appearance models for occlusion handling. In: Int. Work. on Performance Evaluation of Tracking Systems. (2001) 